\title{
Clivage social et appropriation musicale à l'île Rodrigues. Le cas du ségakordéon
}

\section{Guillaume Samson}

\section{(2) OpenEdition}

\section{Journals}

Édition électronique

URL : http://journals.openedition.org/ethnomusicologie/703

ISSN : 2235-7688

Éditeur

ADEM - Ateliers d'ethnomusicologie

Édition imprimée

Date de publication : 1 janvier 2001

Pagination : 163-178

ISBN : 2-8257-0723-6

ISSN : 1662-372X

\section{Référence électronique}

Guillaume Samson, «Clivage social et appropriation musicale à l'île Rodrigues. Le cas du ségakordéon

», Cahiers d'ethnomusicologie [En ligne], 13 | 2001, mis en ligne le 09 janvier 2012, consulté le 01 mai

2019. URL : http://journals.openedition.org/ethnomusicologie/703

Ce document a été généré automatiquement le 1 mai 2019.

Tous droits réservés 


\title{
Clivage social et appropriation musicale à l'île Rodrigues. Le cas du ségakordéon
}

\author{
Guillaume Samson
}

1 Constituant, avec La Réunion et l'île Maurice, l'archipel des Mascareignes, l'île Rodrigues compte, sur un territoire de $144 \mathrm{~km}^{2}$, environ 36000 habitants, soit $3 \%$ de la population totale de la République de Maurice à laquelle elle est rattachée administrativement et politiquement depuis l'indépendance proclamée en $1968^{1}$.

2 N'ayant pas connu au xix siècle le développement d'une monoculture sucrière, caractéristique des sociétés de plantation à Maurice et à La Réunion, et son corollaire qu'ont été les immigrations massives d'engagés indiens, sa population est demeurée en majorité créole, «au sens mauricien du terme qui se définit, en gros, par l'exclusion des catégories ethniques blanche et indienne et la pratique du catholicisme » (Chaudenson 1981 : 117). Ce trait particulier de peuplement qui donne à l'île un caractère à première vue africain (Benoist 1978 :1868) lui confère par ailleurs, vu son isolement historique et son développement économique encore récent, l'image d'une île culturellement " préservée », particulièrement représentative de la culture créole originelle ${ }^{2}$.

Cette image est aujourd'hui elle-même véhiculée par les groupes de musiques folkloriques rodriguais qui profitent du récent développement touristique de l'île pour prendre progressivement leur indépendance vis-à-vis du pouvoir politique local qui les a vu naître et à qui ils servirent de porte-parole culturel dans les années 1970, période d'éveil identitaire et politique non seulement de l'île Rodrigues mais également de La Réunion, de Maurice et des Seychelles.

4 Les enquêtes et séances d'enregistrements menées à Rodrigues en 1978 par Claudie Marcel-Dubois et Maguy Pichonnet-Andral pour le Musée National des Arts et Traditions Populaires $^{3}$ se déroulèrent dans ce contexte nouveau. L'heure était alors à la découverte et à la sauvegarde d'un patrimoine menacé par les musiques internationales, démarche qui intéressait particulièrement les élites locales œuvrant alors pour la reconnaissance 
d'une identité spécifiquement rodriguaise, censée légitimer une position politique à part entière de l'île dans l'État mauricien. Mais ces enquêtes ne donnèrent, hélas, pas lieu à des publications, et les quelques descriptions effectuées depuis sur le monde musical rodriguais furent le fait de chercheurs non musicologues (Chaudenson, Barat) qui se limitèrent en conséquence à des caractérisations générales marquées par l'identification parcellaire des origines et de l'authenticité musicale rodriguaise.

5 Je me propose, au cours de cet article, d'aller plus loin en montrant comment la traditionnelle bi-musicalité rodriguaise a donné lieu à de multiples échanges entre les genres et les répertoires, aboutissant à la constitution du ségakordéon qui emprunte, d'un côté, au séga, genre communément identifié comme étant d'origine africaine et, de l'autre, aux musiques d'accordéon d'origine européenne. On questionnera à cet effet la notion de métissage telle qu'appliquée à cette forme musicale en essayant particulièrement de comprendre pourquoi le terme séga a été adopté et comment les transformations qui s'en suivirent se sont intégrées dans des ordres référentiels plus anciens.

\section{Une population créole : histoire et groupes sociaux}

Découverte en 1528 par le Portugais Diego Rodriguez (North-Coombes 1971: 19), l'île Rodrigues n'a vraiment été colonisée qu'à partir de 1792 avec l'arrivée de colons venus accompagnés de leurs esclaves de l'île de France (l'actuelle île Maurice) et de Bourbon (l'actuelle Réunion). « En 1804, on note ainsi la présence de cinq familles européennes (22 blancs dont 13 appartiennent à la famille Rochetaing, celui-ci étant venu avec Marie, "mulâtresse libre» et sa fille également mulâtresse libre) et 82 esclaves (32 du Mozambique, 18 de Madagascar, 5 Talingas, 1 Guinéen, 1 Malais, 1 Bengali et 24 nés à Rodrigues). On dénombrait donc à cette date 104 personnes dont 82 esclaves. Les catégories au sein desquelles sont rangés les esclaves ne prouvent en aucune façon une origine étrangère; ces esclaves venaient, sans aucun doute, des îles de France et de La Réunion et ce sont eux qui comme certains colons, ont introduit les parlers créoles des autres Mascareignes » (Barat $1985: 18$ ).

7 En 1809, les Anglais prirent possession de Rodrigues d'où ils lancèrent leur offensive pour envahir l'île de France en décembre 1810. Et en 1814, suite au traité de Paris, la France céda l'île de France et Rodrigues à l'Angleterre. D'après Dupon, « la véritable colonisation commence à cette époque [...] et est le fait d'agriculteurs et de pêcheurs mauriciens employant une petite main d'œuvre servile » (1969: 6). Entre 1826 et 1851 la population de l'île quadrupla, passant de 123 à 495 habitants, en raison notamment de l'arrivée de plusieurs colons, blancs et métis, venus de Maurice (Corne et Stein 1979: 60). Sur les 495 habitants recensés en 1851, on compte alors « une cinquantaine de descendants de colons et d'esclaves venus des îles de France et de Bourbon dans la décennie précédente, une centaine de pêcheurs mauriciens installés sur la côte et 350 esclaves malgaches ou africains " (Chaudenson 1992: 34). Par la suite, la population s'est essentiellement développée par accroissement naturel, d'autant que l'île est restée isolée jusqu'à une époque assez récente ${ }^{4}$. Aujourd'hui, la population rodriguaise se répartit schématiquement en deux groupes distincts et somme toute assez endogames :

1. Les noirs, majoritaires, d'origine malgache et africaine, situés surtout dans le centre plus montagneux de l'île, ce qui leur vaut notamment le surnom de «montagnards». Historiquement, leurs activités économiques principales sont l'agriculture et l'élevage. 
2. Les milat (du mot mulâtre) qui sont des « métis d'Européens, d'Africains, en général de teint clair» (Barat 1985 : 23) occupant essentiellement le littoral nord. Traditionnellement, ils sont surtout pêcheurs.

3. On compte aussi quelques descendants d'émigrants indiens et chinois venus au début du siècle pour le petit commerce de la mercerie.

8 Cette répartition de la population trouve ses origines au milieu du xix ${ }^{\mathrm{e}}$ siècle quand, suite à l'abolition de l'esclavage proclamée en 1833, les esclaves libérés allèrent peupler le centre de l'île tandis que les blancs et les métis, d'origine mauricienne plus récente pour certains, développaient sur la côte des activités de pêche et de commerce (NorthCoombes 1971 : 79). Pratiquant jusqu'à la fin des années 1960 une agriculture et une pêche d'autosubsistance, l'île devint ensuite de plus en plus dépendante de sa métropole suite à plusieurs périodes de sécheresse et cyclones dévastateurs. A partir des années 1980, l'État mauricien engagea ainsi un nombre croissant de Rodriguais à des travaux d'aménagement du territoire, devenant de loin le plus gros employeur de l'île.

9 Ce bref rappel historique révèle un paradoxe marquant de la situation sociale et culturelle rodriguaise. Rodrigues, « où les grandes plantations ne trouvaient pas de conditions propices» (Benoist 1978: 1867), et qui, en conséquence, demeura exclusivement paysanne, n'a pas directement connu les bouleversements économiques, sociaux et culturels qui ont accompagné, dans les deux autres Mascareignes, le passage d'une société "d'habitation» à une société «de plantation». L'histoire de son peuplement est toutefois complètement liée à cette période transitoire qu'est la fin du xviii ${ }^{\mathrm{e}}$ siècle où, à La Réunion comme à Maurice, on est passé d'un mode de vie colonial paysan (adaptation au milieu, difficultés de survie, polyculture vivrière, faible concentration des terres) au sein duquel les maîtres entretenaient avec leurs esclaves, moins nombreux, une proximité quotidienne, à un système d'exploitation agroindustrielle (monoculture extensive, esclavagisme, et plus tard engagisme indien de grande ampleur) où la supériorité numérique écrasante des esclaves sur les maîtres consacrait désormais la division des espaces de vie. Les acquis linguistiques et culturels véhiculés par les colons et les esclaves venant de Maurice et de La Réunion trouvèrent malgré tout à Rodrigues les conditions favorables à leur développement. Ainsi, dès le début de la colonisation, le nombre d'esclaves par famille fut supérieur à celui des maîtres 5 .

10 Ce fait significatif a pu se traduire très tôt par l'émergence d'un répertoire musical commun à tous les esclaves, lesquels se constituèrent après l'abolition de l'esclavage en groupe social à part entière occupant un espace géographique déterminé. Selon toute vraisemblance, les formes premières du séga rodriguais se seraient dès lors construites à partir d'éléments musicaux propres, à l'époque, au séga mauricien et à ce que l'on nomme aujourd'hui maloya à La Réunion. Les affinités musicales que l'on peut aujourd'hui constater entre ces musiques (polyrythmies ternaires, importance des membranophones et des idiophones, forme responsoriale des chants) permettent en effet de postuler l'existence d'une sorte de fonds musical commun à ces trois îles.

\section{Une tradition bi-musicale}

11 Les premiers témoignages écrits sur les pratiques musicales en cours à Rodrigues remontent à 1863 sous la plume du Père Pivault, qui note un clivage musical caractéristique entre les populations noires de la Montagne et celle de la capitale côtière : 

deux genres musicaux différents qui, corollairement aux origines des groupes ethniques qui constituent la population, sont désignés communément comme étant, l'un d'origine européenne et l'autre d'origine africaine ${ }^{6}$. Le premier comprend aujourd'hui les musiques de danse jouées à l'accordéon, qui supplanta le violon au début du $\mathrm{xx}^{\mathrm{e}}$ siècle : polka, mazok (mazurka), kotis (scottish), quadrille, laval (valse), biguine, one step, raspa; tandis que le second constitue le séga sous ses différentes formes traditionnelles : séga plant zariko (séga pour planter les haricots), séga tanbour (séga au tambour) et ségakordéon (séga à l'accordéon), ce dernier ayant un statut particulier puisqu'on le jouait au cours du quadrille. Ces deux genres musicaux ne sont à l'heure actuelle plus pratiqués dans leurs contextes traditionnels et sont essentiellement liés à l'activité de groupes folkloriques qui, pour des raisons scéniques, ont partiellement laissé de côté certains répertoires anciens comme les romances, exécutées autrefois dans les mariages, et les séga plant zariko qui étaient liés aux travaux des champs.

13 Cependant, si l'habitude et la commodité veulent que l'on désigne d'abord les différentes traditions de l'île en termes de « dichotomie d'origine » (Lafontaine 1988a : 23), il importe également de prendre en compte les processus transculturels qui font que ces musiques sont aujourd'hui des formes autonomes et que ceux qui les pratiquent les considèrent comme étant authentiquement rodriguaises. Après avoir identifié en Guadeloupe une situation presque analogue, Marie-Céline Lafontaine émet les hypothèses suivantes :

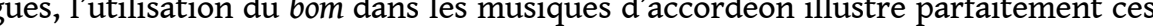
interférences entre les systèmes organologiques africains et européens. Cet instrument est un arc musical, d'environ 1,60 m de haut, avec résonateur en calebasse ou en noix de coco. Le musicien en joue assis sur une caisse de bois sur laquelle il pose par intermittence l'extrémité inférieure de son arc, ce qui lui procure un deuxième résonateur en plus de la calebasse. On trouve également cet instrument, mais sans la caisse, à La Réunion et aux Seychelles ainsi qu'à Madagascar et au Mozambique. 
Fig. 1 : Le groupe Ziskakan (" Jusqu'à quand ? ») en représentation à l'Hôtel Mourouk, Pointe Corail, île Rodrigues.

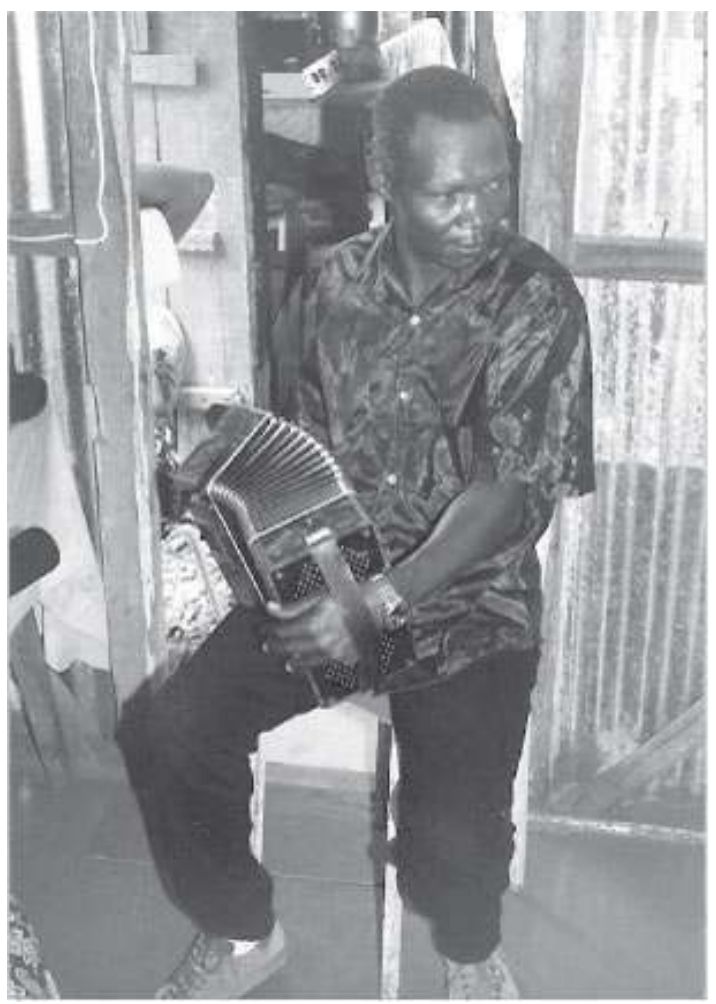

Photo : Guillaume Samson, 1996

Fig. 2 : L'accordéoniste Yom, un après-midi de divertissement à Fond Grand-Baie, île Rodrigues.

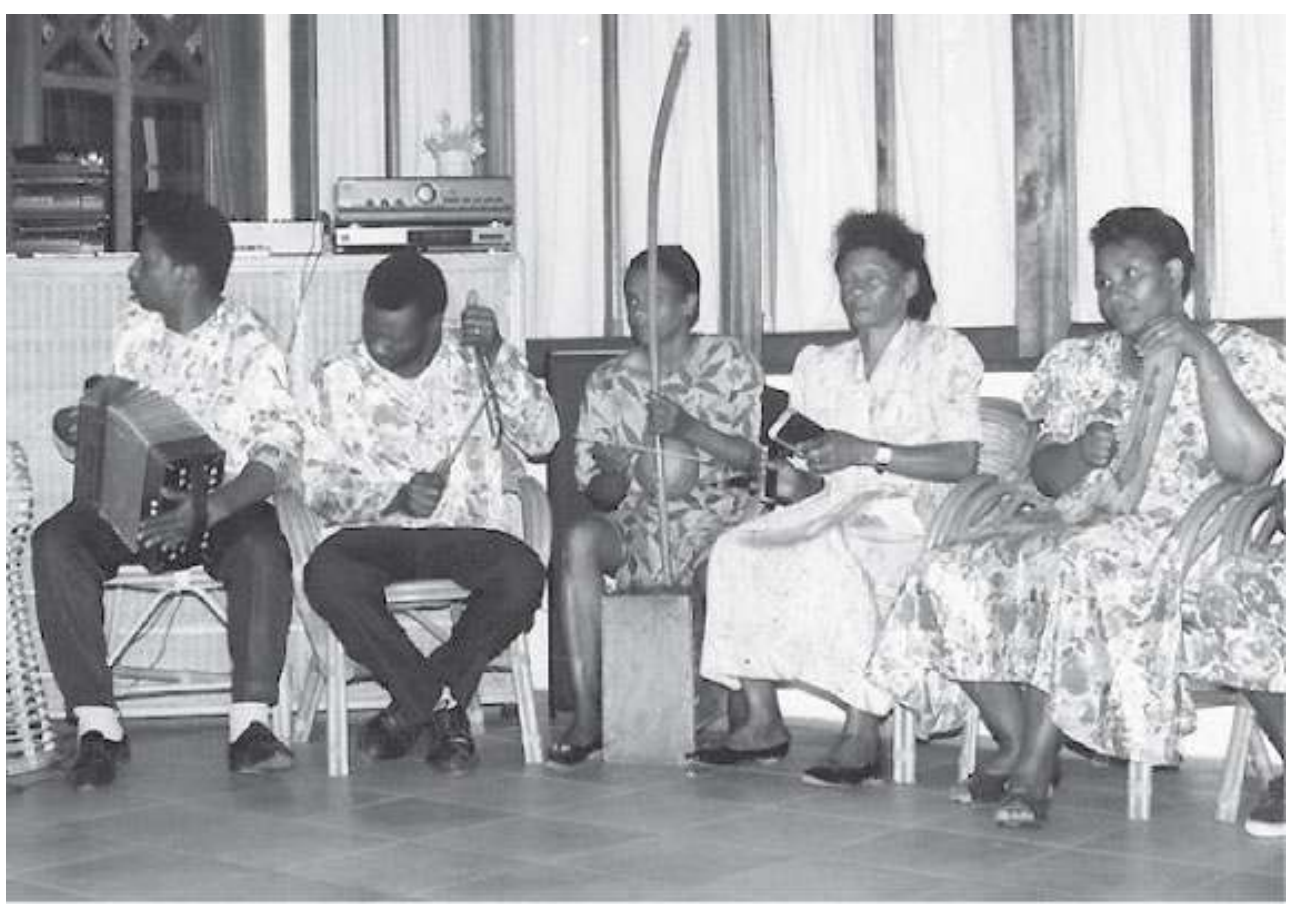

Photo : Guillaume Samson, 1996. 
Toutefois, les interférences entre musiques européennes et séga sont plus profondes que ces simples emprunts et les « syncrétismes » entre diverses cultures musicales africaines semblent difficiles à mettre au jour de façon précise à Rodrigues vu le caractère controversé de l'origine ethnique précise des esclaves. Malgré tout, la forme responsoriale et/ou antiphonale de nombreux chants, le soubassement pentatonique de certains, l'importance de la pulsation, de la répétition et de la polyrythmie, caractéristiques marquantes des musiques africaines (Arom), définissent bien le caractère plutôt africain du séga. L'intérêt est dès lors de dégager, au-delà de cette tendance générale, les processus qui ont sous-tendu l'autonomisation de ces chants. S'ils sont aujourd'hui des «objets constitués » (Bastide 1996), rendre compte de leur dynamisme dépasse donc la question du métissage formel (en tant que fusion, ou superposition, d'une musique avec une autre). Plus que la nature des emprunts à telle ou telle culture musicale, c'est la propension à participer ou non de transformations sociales et à matérialiser « un mode d'expérience » (Geertz 1986) qui importe. Les différentes formes prises par le séga rodriguais et les rapports qu'elles entretiennent entre elles constituent à cet effet un indicateur marquant des remaniements identitaires opérés à travers la musique.

\section{Typologie des séga}

\section{Séga plant zariko}

Les séga plant zariko étaient des chants de travail saisonniers liés à l'activité agricole, et plus particulièrement à la culture des haricots. Leur apparition n'est pas datée, mais ils furent chantés jusqu'à la fin des années 1960 et restent aujourd'hui dans le souvenir de nombreuses personnes âgées. On les exécutait exclusivement dans les champs familiaux ${ }^{7}$ dont l'exploitation donnait lieu à un système d'entraide collective entre voisins et parents. Composés d'une courte mélodie syllabique répétée en forme responsoriale, ils sont construits sur l'échelle diatonique, variant les couleurs majeures ou mineures et reproduisant parfois de façon sous-jacente des profils mélodiques pentatoniques. Leur exécution était rythmée par le choc des pioches frappant le sol lors de la plantation et celui des bâtons que l'on utilisait pour l'égrenage des haricots. Dans les deux cas, l'accompagnement rythmique produit par entrecroisement fournissait un cadre binaire. Les textes, circonstanciés et souvent satiriques, étaient soit improvisés sur place, soit choisis dans un répertoire préexistant, et la prédominance des femmes dans les affaires agricoles explique sans doute le rôle de solistes qu'elles tenaient, apparemment plus fréquemment que les hommes, dans la conduite de ces chants. Ce rôle musical était doublé d'un rôle social important car c'étaient-elles qui improvisaient les textes ou choisissaient de chanter tel ou tel séga connu de tous et déjà porteur de sens.

\section{Séga tanbour}

17 Les séga tanbour sont des chants festifs que l'on pratiquait autrefois lors de soirées villageoises qui portaient le même nom. Les textes et la danse y avaient un rôle central. Reprenant la présentation que donne Lafontaine du léwoz guadeloupéen (1988b : 83), on dira pareillement que le séga tanbour, par ses textes anecdotiques et circonstanciés, était le «lieu où se régl(ai)ent les conflits interindividuels et où peuvent s'exprimer les 
tensions qui travers(ai)ent la société globale ». Constitués d'une seule et courte mélodie syllabique, ils étaient exécutés en forme responsoriale par une soliste appelée " composeur », qui improvisait ou décidait de réactualiser tel ou tel séga déjà porteur de sens pour l'auditoire, et un chœur composé du reste des participants. L'accompagnement rythmique est produit par deux tanbour (membranophones cylindriques à peau collée enrichis de trois paires de sonnailles latérales) et d'idiophones variés (bâtons ou boîtes de conserves entrechoqués, mains). Ceux-ci exécutent un cadre rythmique répétitif dont la cellule de base peut approximativement être définie comme étant à deux temps ternaires. L'échelle utilisée est identique à celle des séga plant zariko.

\section{Ségakordéon}

18 Les ségakordéon étaient joués autrefois lors de bals privés, réservés essentiellement à la pratique des danses occidentales, appelés bal rand zariko ou bal lé roi («bal les rois») conformément à la pratique de réciprocité qui y avait cours. Les ségakordéon constituaient dans ce contexte la dernière figure du quadrille. L'instrument central est l'accordéon diatonique qui, avec l'harmonica dans une moindre mesure, demeure le seul aérophone intégré à la musique traditionnelle rodriguaise. L'accordéoniste en joue exclusivement assis en posant son instrument sur la cuisse. L'accompagnement rythmique est habituellement produit par une caisse en bois plus ou moins épais, utilisée uniquement dans ce contexte-là par le joueur de bom qui laisse de côté son arc musical et frappe les parois de son instrument des deux mains, produisant des rythmes entrecroisés qui imitent ceux du séga tanbour. Il est également soutenu par deux triangles, généralement de grande taille, et des idiophones divers (boîtes, bâtons, planchettes de bois entrechoquées, hochets et sonnailles de création plus ou moins récente). Composée de plusieurs mélodies courtes et de segments mélodico-rythmiques agencés selon un ordre de répétition variable laissé à l'appréciation du musicien, l'organisation mélodique générale des ségakordéon est la plus complexe des séga. L'échelle utilisée y est essentiellement diatonique et les mélodies sont développées dans les modes majeurs. Si aujourd'hui il semble que ce sont des formes autonomes (on peut jouer un ségakordéon sans forcément jouer le quadrille), elles appartiennent à la fois aux musiques d'accordéon et au séga.

\section{Transculturalité et identité musicale}

19 D'emblée, on constate que ces trois formes de séga présentent entre elles d'importantes différences musicales et contextuelles qui posent directement la question des fondements $\mathrm{du}$ séga comme genre musical. Si celui-ci possède une identité particulière, où se situe-telle? Au niveau de la musique, des textes, de l'histoire de ses différents contextes d'exécution? Quels critères ont pu guider l'attribution du même terme à ces différentes pratiques?

20 Musicalement, les séga plant zariko et le séga tanbour s'opposent rythmiquement (binaire/ ternaire) mais leurs formes mélodiques présentent d'importantes analogies (une seule mélodie exécutée en forme responsoriale). Séga tanbour et ségakordéon sont, quant à eux, très proches du point de vue rythmique (formule polyrythmique à deux temps ternaires), mais diffèrent au niveau de la mélodie (une seule mélodie / plusieurs mélodies; monophonie / polyphonie) et des modalités d'exécution. Ces rapports analogiques et 
antinomiques variés rendent difficile la définition du séga comme un phénomène musical structuré dont l'examen synchronique des lois internes permettrait de résoudre les apparentes contradictions.

$21 \mathrm{Au}$ vu de l'histoire complexe du terme séga dans les Mascareignes, son attribution aux différentes pratiques auxquelles il a renvoyé à Rodrigues semble au contraire s'être effectuée suivant des dynamiques de transferts variées, le mot faisant référence, selon les époques et les circonstances, tantôt à des éléments d'ordre social et contextuel, tantôt à des éléments d'ordre musical. Apparu à Maurice à la fin du xviiie siècle sous la forme " tshiéga» (Chaudenson 1995: 111), il désigne en 1817, d'après le témoignage de L. de Freycinet, deux musiques différentes pratiquées par les noirs :

Cette double signification, qui englobe ici des formes musicales de type tant européen qu'africain - l'identité réellement mozambicaine de cette dernière, qui n'est d'ailleurs pas sans rappeler la description du séga à Rodrigues du Père Pivault, étant, comme celle des esclaves, à remettre en cause ou au moins à vérifier - fait donc référence à une catégorie socio-ethnique. Le "tchéga», c'est d'abord la musique des noirs, sans différenciation de contexte. Pour l'île Rodrigues, où les esclaves vinrent avec leur maîtres de La Réunion et de Maurice, c'est cette explication qui semble la plus apte à éclairer, au départ, les différences entre séga plant zariko et séga tanbour, une pareille situation facilitant cependant les échanges et les emprunts.

Le ségakordéon marque une rupture avec cet état de fait. La référence directe au rythme et à la danse du séga tanbour et son intégration dans le contexte des musiques d'accordéon, d'abord associées aux catégories blanches et métisses de la population, laissent à penser qu'il fut l'œuvre des populations noires de la Montagne quand elles se les approprièrent, selon toute vraisemblance au début $\mathrm{du} \mathrm{xx}^{\mathrm{e}}$ siècle, en reconnaissant par-là implicitement la nouveauté et la différence par rapport à leur culture musicale première. Les danses d'accordéon devenues elles aussi danses des Noirs, le sens du terme séga se serait précisé et aurait désigné, par la fixation du critère rythmique, « la musique et la danse que les Noirs pratiquaient avant», "leur tradition musicale ancienne». La dénomination complète des différents séga traduit également cette importance nouvelle des éléments musicaux: séga plant zariko (séga pour planter les haricots) renvoie directement au contexte d'exécution spécifique de cette musique, tandis que séga tanbour (" séga au tanbour ») et ségakordéon ("séga à l'accordéon ») font d'abord référence à l'instrument central de leur orchestration et seulement en deuxième instance à une appartenance contextuelle particulière.

Le ségakordéon constitue donc une sorte de marqueur inversé de ce processus de remaniement musical et identitaire, et fonde, indirectement, la constitution du séga comme un genre musical doté de caractéristiques formelles qui lui sont propres, indépendamment des données ethniques.

Un examen musicologique précis devra préalablement nous renseigner sur sa forme générale, sa dynamique et ses normes esthétiques. On s'attachera ensuite à en préciser les conditions d'exécution afin de mieux cerner la place symbolique tenue par cette musique.

\section{Le ségakordéon : forme et structure}

Un ségakordéon est habituellement précédé d'une forme d'appel, les accordéonistes et les joueurs de triangle (tryangèr) faisant sonner leur instrument de manière saccadée et 
brisée, comme s'ils commençaient à jouer, puis s'arrêtaient aussitôt. Cette pratique se retrouve également chez les joueurs de tanbour (tanbouryé) dans le séga tanbour.

Quand l'accordéoniste, suivi aussitôt par les tryangèr, débute la pièce en tant que telle, entrent alors les autres idiophones et la caisse qui produisent une polyrythmie ternaire continue. On répétera ensuite sans interruption plusieurs mélodies courtes (de deux à cinq en moyenne), basées sur le schéma occidental tonique-dominante. L'agencement des mélodies est laissé au libre choix de l'accordéoniste qui en varie l'occurrence à son gré au cours de l'exécution. Pour annoncer la fin du séga, l'accordéoniste répète une ou deux formules mélodico-rythmiques ascendantes courtes qui créent un effet de suspension.

L'accordéon, qui constitue à lui seul un instrument polyphonique, introduit par ailleurs l'harmonie dans le séga, non seulement parce que le musicien peut jouer un accompagnement de basses et d'accords mais aussi parce qu'il peut doubler la mélodie. Le rôle des basses et des accords y semble toutefois plus rythmique que réellement harmonique. Chez les accordéonistes rodriguais, et surtout chez les plus âgés, l'utilisation de l'accompagnement fait en effet l'objet de transgressions harmoniques et d'adaptations personnelles plus ou moins marquées dues, semble-t-il, à une triple contradiction entre les limites harmoniques de l'instrument, les contraintes imposées par la mélodie et le rôle rythmique important assigné au jeu des basses et des accords. Il en résulte, pour l'auditeur occidental inaccoutumé, une forte impression de dissonance qui peut être accentuée par un jeu de triangle véhément.

En outre, les ségakordéon donnent parfois lieu à des parties chantées, avec ou sans paroles, à l'unisson avec l'accordéon qui font apparaitre des dialogues de type question-réponse rappelant la forme responsoriale des séga tanbour et des séga plant zariko. La présence de paroles se comprend assez bien si l'on considère, d'une part, que le répertoire d'accordéon à l'intérieur duquel s'insère également le ségakordéon est constitué essentiellement de chansons, bien que les musiciens en chantent également rarement les paroles, et, d'autre part, que les textes sont un élément permanent dans tous les autres types de séga.

\section{Évaluation des processus de métissage}

Métaphore du biologique selon Jean Benoist, le concept de métissage, appliqué en anthropologie, est chargé idéologiquement. Comme ceux d'acculturation et de syncrétisme, il décrit indirectement un «état » (Benoist 1996: 56) et place les processus qu'il sous-entend au centre d'une identité conflictuelle, à part, basée sur une rupture des « essences originelles » (Benoist 1992, 1996). Si le métissage est mélange, il implique en effet l'identification de cultures fondatrices implicitement reconnues comme pures.

31 Or, dans notre cas, les innovations caractéristiques apportées par le ségakordéon ne rentrent dans ce cadre conceptuel qu'au prix d'une réduction éculée qui accorde d'emblée, à l'Afrique, les innovations rythmiques et, à l'Europe, les inventions mélodiques, la fusion des deux offrant un produit musical nouveau et créatif. À l'île Rodrigues, la coexistence exclusive de ces deux composantes musicales, avec des correspondances organologiques distinctives, renforce d'autant plus cette tentation. Prolongeant ce raisonnement, le ségakordéon serait né de la rencontre du rythme du séga tanbour avec les mélodies de l'accordéon. La prédilection pour les modes majeurs, alors que les mélodies des séga tanbour et des séga plant zariko présentent une plus grande 
variété de couleurs mélodiques, fait ainsi référence aux musiques d'accordéons, l'accompagnement rythmique et la tendance marquée à la polyrythmie du jeu des basses de l'instrument rappelant, en revanche, directement les entrecroisements rythmiques du séga tanbour. Toutefois, ces innovations musicales entretiennent un rapport dialectique étroit, conférant au ségakordéon une homogénéité structurelle qui lui fait dépasser le stade d'« hybride» (Kartomi 1981 : 232, 233). La pertinence du débat sur les origines en est par là amoindrie. D'une part, les mélodies des séga plant zariko et des séga tanbour, musiques dont on a noté le caractère plutôt africain, suivent parfois un contour typiquement occidental. D'autre part, le caractère non exclusif des répertoires du séga rend d'autant plus douteux leur quelconque provenance. Il est ainsi possible d'entendre des groupes folkloriques exécuter des séga plant zariko avec un accompagnement de séga tanbour, ou encore des mélodies de séga moderne mauriciens à la mode jouées en ségakordéon, le rythme et la justesse de la mélodie changeant évidemment en fonction de l'accompagnement utilisé.

Toutes les formes de séga participent donc en quelque sorte du métissage ou du transculturel, à des degrés et des niveaux certes différents, et le ségakordéon n'est pas opposé par nature à ses prédécesseurs. Sa particularité fut cependant de réaliser explicitement un rapprochement entre séga et musiques d'accordéon, entre la musique des «noirs» et celle des blancs et des métis. Et si son appellation évoque bien cette double appartenance musicale, elle interroge par ailleurs les motivations et les modalités sociales de cette nouvelle proximité.

\section{Les bals d'accordéon}

33 On présume que la présence de l'accordéon dans la musique rodriguaise remonte à la fin du xix ${ }^{e}$ siècle ou au début du xxe siècle (La Selve 1995 : 208). La constitution du ségakordéon serait donc quelque peu postérieure à cette période, en raison du temps qu'il a sans doute fallu à la pratique de cet instrument pour pénétrer majoritairement le monde de la «montagne », lieu du séga tanbour, et y devenir réellement populaire. Quoi qu'il en soit, l'accordéon semble avoir tenu assez tôt une place importante dans la vie musicale rodriguaise : il servait notamment, lors des mariages, à conduire le cortège et à animer le bal. Mais les ségakordéon proprement dits se pratiquaient essentiellement au cours des bal lé roi, soirées dansantes dont il était l'instrument central ; celles-ci s'appelaient également bal lakordéon (bal à l'accordéon). Il est probable qu'une partie des musiques qui y étaient alors exécutées aient été apportées par les émigrants rodriguais partis travailler à $\mathrm{La}$ Réunion dans les années 1930 (La Selve 1995 : 218).

Comme les séga tanbour, les bal lakordéon se déroulaient chez les particuliers. On y comptait théoriquement, en plus des musiciens, au minimum seize personnes réparties en huit couples (Chaudenson 1981: 135), correspondant au nombre nécessaire de danseurs pour réaliser les figures du quadrille. Les bals avaient toujours lieu à l'intérieur des cases et, à l'instar des séga tanbour, l'étroitesse du cadre imposait une organisation particulière de l'espace musical. Les espaces réservés à la musique, à la danse et à la restauration ne constituaient toutefois pas des zones fermées ; les musiciens se relayaient fréquemment pour prendre part, à leur tour, à la danse et les danseurs fatigués pouvaient aller s'asseoir sur des chaises ou des bancs placés contre les murs. On se restaurait également près de tables disposées à cet effet, le centre de la pièce étant réservé à la danse. 

tous les jours, la chorégraphie du ségakordéon en reproduit par contre trait pour trait les dispositions et les mouvements. Les participants constituent un cercle, appelé « rond » au centre duquel viennent évoluer un ou deux couples de danseurs dont les mouvements mettent en scène l'approche érotique, sans jamais que les partenaires ne se touchent. $\mathrm{Au}$ cours de la danse, ils se font remplacer successivement par ceux et celles qui, en attendant leur tour, constituent le rond. Les pas consistent en des piétinements resserrés, un des deux pieds étant légèrement en avant par rapport à l'autre qui semble plus mobile, et les déhanchements des danseurs se prolongent en balancements, tours et cambrures variés. On note par ailleurs cette position caractéristique qui consiste chez les femmes à tenir, d'une main, le bout de leur robe ainsi soulevée d'un côté tandis que les hommes peuvent positionner successivement de plusieurs manières leur bras : soit écartés en croix, soit en demi-cercle autour de la tête de la danseuse, soit un bras tendu presque à l'horizontale et l'autre replié vers soi. Ces positions étaient, semble-t-il, censées protéger le couple d'une éventuelle « coupe » effectuée par un membre ou un couple se détachant du rond. Cette façon de danser face à face se nomme baré. La Selve $(1995: 214)$ mentionne la dénomination séga-coupé pour désigner le changement forcé de partenaire. quelque sorte, les attaquaient, doit avant tout être vu comme une mise en scène: on jouait à protéger sa partenaire mais on savait très bien qu'à un certain moment un autre 
danseur viendrait heureusement prendre notre place. Le rythme très rapide du séga ne permettait pas que les danseurs demeurent longtemps dans le rond et être "coupé " était, au bout d'un certain temps, salvateur pour le danseur ou la danseuse.

Dernière figure du quadrille, la danse du ségakordéon marque une rupture symbolique avec les autres danses d'accordéon dont la convenance chorégraphique et le contexte d'exécution formalisé contrastaient avec le séga tanbour et semblaient même s'y opposer. La coexistence de deux types de bal au sein des populations noires de la campagne ne confère donc pas au ségakordéon un statut de réminiscence, plus ou moins volontaire, de musiques délaissées mais l'inscrit bien comme participant d'un positionnement identitaire.

\section{Transferts musico-sémantiques et constructions identitaires}

Par sa position intermédiaire, le ségakordéon révèle la pluriréférentialité et le dynamisme inhérents au terme séga.En effet, les motivations et enjeux qui sous-tendaient la pratique des séga traditionnels rodriguais ne convergent pas, par-delà leur diversité, vers un fondement commun. Lors de l'exécution des séga plant zariko, c'est avant tout le bon déroulement de la collaboration saisonnière qui était l'enjeu central, cette pratique musicale étant fortement liée à son contexte agricole. La critique sociale et la danse étaient en revanche les motivations principales des séga tanbour, organisés spécifiquement à ces fins. Quant aux ségakordéon, ils s'inséraient dans le contexte plus général de bals où la danse et l'entretien des processus d'échange entre les individus donnaient lieu à des pratiques assez formalisées. De même, l'importance moindre accordée aux textes dans le ségakordéon et leur aspect beaucoup moins satirique ôte à cette pratique du séga toute fonction de révélateur social.

Ces données ouvrent d'intéressantes perspectives concernant, d'une part, les sens successifs du mot séga et d'autre part les modalités cognitives de leur usage simultané. Une approche diachronique telle qu'on l'a esquissée permet en effet d'appréhender les stratégies d'usage dont il a pu faire l'objet, tant en terme d'évolution linéaire (passage du critère ethnique au critère musical d'appartenance) que de synchronies successives (expression du clivage ancien noir/blanc qui n'est plus musical mais qui perdure socialement).

Les transferts sémantiques sur lesquels se fonde la cohérence du séga en tant que genre musical confirment donc la nature éminemment historique de ces formes culturelles qui se dérobent souvent devant les caractérisations univoques. Le séga fut en cela d'autant plus susceptible d'assumer des manipulations symboliques multiples selon les circonstances et les catégories sociales concernées.

Et si les mouvements identitaires de ces vingt-cinq dernières années qui, dans les Mascareignes, virent le développement du revivalisme, ont parfois figé les formes culturelles, leur histoire n'en n'éclaire pas moins certains processus récents de fusion musicale. Ainsi, au début des années 1990, des musiciens mauriciens, issus en majorité de la catégorie créole de la population et engagés dans une recherche d'Africanité, inventèrent le seggae, à partir du séga moderne et du reggae. Ils réinterprétèrent les termes de cette nouvelle configuration musico-sémantique, le séga représentant désormais l'identité locale tandis que le reggae symbolisait le retour à l'Afrique. Le seggae, 
qu'accompagnait un ensemble d'autres codes visuels (coiffure, couleurs rastafariennes), langagiers, gestuels, et des textes engagés, connut un franc succès aux Mascareignes et aux Seychelles, donnant lieu à d'autres adaptations et manipulations qui, cependant, ne remirent pas en cause sa configuration musicale de base (Samson 1998).

Contrairement aux apparences et à certains préjugés, la mise au jour des identités musicales et la définition des répertoires concernent donc particulièrement l'ethnomusicologie des musiques dites "métisses", malgré les réserves que l'on peut émettre sur l'utilisation de ce terme. Si cette entreprise peut parfois paraître fastidieuse en raison du caractère fuyant des musiques qu'elle aborde, elle oblige cependant à dépasser le discours qui se pose en terme de "dichotomie d'origine » sans toutefois nier l'existence de cette rencontre récente de cultures musicales auparavant étrangères l'une à l'autre. En cherchant à mettre au jour les fondements d'ordres référentiels sans cesse renouvelés, l'occasion est ainsi offerte à l'ethnomusicologue d'opérer ce lien fructueux et tant recherché entre le musical et le social, ni l'un ni l'autre de ces domaines ne se suffisant à lui-même comme élément d'explication. Au contraire seule la conjugaison habile et complémentaire des deux semble pouvoir rendre compréhensible une situation complexe de contact de culture.

\section{NOTES}

1. Rodrigues est la $21^{\mathrm{e}}$ circonscription administrative de l'île Maurice.

2. Dupon écrit à ce titre: "elle présente les traits les moins altérés de cette culture créole ancienne que les influences plus récentes et plus puissantes ont étouffé à La Réunion et à l'Ile Maurice ». (Dupon 1977 : 1105).

3. Mascareignes Juillet-Aout 1978, Mission CNRS-Université de La Réunion.

4. Jusque dans les années 1960, Rodrigues était ainsi reliée à Maurice environ une fois par mois par bateau. Dans les années 1970, la fréquence des voyages s'est intensifiée jusqu'à atteindre aujourd'hui 28 aller-retours par an. On a également construit un aéroport qui accueille aujourd'hui plusieurs fois par jour un avion d'Air Mauritius, favorisant le développement du tourisme. Par ailleurs, les télécommunications n'ont cessé de se développer depuis ces vingt dernières années, rapprochant désormais Rodrigues de sa métropole. Ces nouvelles données vont de pair avec l'insertion de Rodrigues dans des réseaux internationaux (coopération internationale (FED), mouvements de jeunesse (JOC), ONG, tourisme international).

5. En 1804, il ne reste sur l'île que cinq familles qui possèdent un nombre assez conséquent d'esclaves. Marragon possède 37 esclaves pour 100 arpents cultivés. Le Gros en posséde 6 pour 25 arpents, Gorry 19 pour 70 arpents, et Rochetaing 19 pour 70 arpents.

6. Chaudenson remarque à ce propos: «Le point intéressant pour le système culturel que constitue la musique créole est que se sont maintenues, en dépit de multiples échanges et interférences, deux traditions musicales : l'une à dominante européenne, celle des blancs ou plus précisément des classes dominantes, mulâtres compris, car le clivage n'est pas seulement ethnique, l'autre à dominante non européenne, généralement africaine, celle des noirs. L'illustration de cette dualité se trouve sans doute à Rodrigues où la population est pourtant exclusivement noire ou mulâtre. » (1995 : 114) 
7. Ce domaine d'activité est resté longtemps celui des femmes et des enfants, excepté, justement, lors des travaux qui nécessitaient une grosse main-d'œuvre tels que la plantation et la récolte de haricots ou de maïs, où l'on faisait appel aux hommes et au voisinage.

8. Cf. infra.

\section{RÉSUMÉS}

A l'Ile Rodrigues, la constitution du ségakordéon semble avoir résolu, en l'exprimant, le conflit symbolique originel entre la musique des noirs, réprésentée par le séga, et celle des métis, jouée à l'accordéon, témoignant, à ce titre, d'un processus identitaire d'appropriation. L'auteur montre, par ce biais, que répondre à la question du métissage musical ne peut se réduire à étudier des configurations culturelles formelles. Il insiste particulièrement sur les stratégies d'usage et de transferts musico-sémantiques qui fondent la cohérence dynamique du séga comme genre musical. En mettant au jour les modalités de passage d'une forme de séga à l'autre, il souligne ainsi la pertinence de l'étude des conceptualisations et des réinterprétations passées dont une meilleure connaissance pourra aider, même sur des terrains voisins, à interpréter des phénomènes plus récents de création musicale.

\section{AUTEUR}

\section{GUILLAUME SAMSON}

Guillaume SAMSON poursuit actuellement des recherches à l'Université de La Réunion (Centre d'étude des Sociétés de l'Océan Indien) sur les rapports entre musiques traditionnelles et musiques modernes dans les Mascareignes. Son mémoire de maîtrise (Université de Nanterre, 1997) traitait, dans une approche comparative, de la diversité du séga à l'Ile Rodrigues. Dans son mémoire de D.E.A. (Université de La Réunion, 1998), il a, par ailleurs, cherché à évaluer la portée théorique de la notion de créolisation musicale à travers l'appropriation du reggae à l'ile de La Réunion. 